\title{
Melatonin-induced DNA demethylation of metal transporters and antioxidant genes alleviates lead stress in radish plants
}

\author{
Mingjia Tang ${ }^{1}$, Liang Xu', Yan Wang ${ }^{1}$, Junhui Dong ${ }^{1}$, Xiaoli Zhang ${ }^{1}$, Kai Wang ${ }^{1}$, Jiali Ying ${ }^{1}$, Cui Li ${ }^{1}$ and Liwang Liu (1)
}

\begin{abstract}
Melatonin (MT) is a tryptophan-derived natural product that plays a vital role in plant response to abiotic stresses, including heavy metals (HMs). However, it remains elusive how exogenous MT mediates lead (Pb) accumulation and detoxification at the methylation and transcriptional levels in radish. In this study, decreased Pb accumulation and increased antioxidant enzyme activity were detected under MT treatment in radish. Single-base resolution maps of DNA methylation under Pb stress (Pb200) and Pb plus MT treatment (Pb_50MT) were first generated. The genomewide methylation level was increased under Pb stress, while an overall loss of DNA methylation was observed under MT treatment. The differentially methylated region (DMR)-associated genes between Pb_50MT and Pb200 were uniquely enriched in ion binding terms, including cation binding, iron ion binding, and transition metal ion binding. Hyper-DMRs between Pb200 and Control exhibited a decreasing trend of methylation under Pb_50MT treatment. A few critical upregulated antioxidant genes (e.g., RsAPX2, RsPOD52 and RsGST) exhibited decreased methylation levels under MT treatment, which enabled the radish plants to scavenge lead-induced reactive oxygen species (ROS) and decrease oxidative stress. Notably, several MT-induced HM transporter genes with low methylation (e.g., RsABCF5, RSYSL7 and RsHMT) and transcription factors (e.g., RsWRKY41 and RSMYB2) were involved in reducing Pb accumulation in radish roots. These findings could facilitate comprehensive elucidation of the molecular mechanism underlying MTmediated $\mathrm{Pb}$ accumulation and detoxification in radish and other root vegetable crops.
\end{abstract}

\section{Introduction}

Contamination of soil and water with heavy metals (HMs) has become an increasingly concerning problem worldwide and affects human health through the food chain $^{1,2}$. Lead $(\mathrm{Pb})$, a widespread HM pollutant, is considered as a carcinogen and does not have any beneficial biological functions among all organisms. Lipid peroxidation, excessive reactive oxygen species (ROS), and DNA damage can be induced by $\mathrm{Pb}$ stress, resulting in the inhibition of plant growth and development ${ }^{3}$. Due to the transfer of $\mathrm{Pb}^{2+}$ from contaminated agricultural soil and

\footnotetext{
Correspondence: Liwang Liu (nauliulw@njau.edu.cn)

${ }^{1}$ National Key Laboratory of Crop Genetics and Germplasm Enhancement, Key Laboratory of Horticultural Crop Biology and Genetic Improvement (East China) of MOAR, College of Horticulture, Nanjing Agricultural University, Nanjing 210095, P.R. China
}

irrigation water to food plants, several vegetable crops take up excess $\mathrm{Pb}^{2+}$ via different pathways, which potentially disrupts the nutrient balance in plants ${ }^{4,5}$. Hence, the reduction of $\mathrm{Pb}$ accumulation is imperative to effectively prevent toxicity in foods from crop plants.

DNA methylation, as an epigenetic modification, is associated with transcriptional activity and gene expres$\operatorname{sion}^{6,7}$. In plant genomes, methylated cytosines are categorized into three contexts: CG, CHG, and $\mathrm{CHH}$ (where $\mathrm{H}=\mathrm{A}, \mathrm{T}$, or C). In Arabidopsis, CG and CHG methylation are maintained by a conserved DNA METHYLTRANSFERASE 1 (MET1) protein and CHROMOMETHYLASE3 (CMT3), respectively. Moreover, CMT2 and domain rearranged methyltransferase 2 (DRM2) primarily maintain $\mathrm{CHH}$ methylation ${ }^{8,9}$. In plants, DNA methylation levels are regulated not only by

\section{(c) The Author(s) 2021}

(c) (i) Open Access This article is licensed under a Creative Commons Attribution 4.0 International License, which permits use, sharing, adaptation, distribution and reproduction c. in any medium or format, as long as you give appropriate credit to the original author(s) and the source, provide a link to the Creative Commons license, and indicate if changes were made. The images or other third party material in this article are included in the article's Creative Commons license, unless indicated otherwise in a credit line to the material. If material is not included in the article's Creative Commons license and your intended use is not permitted by statutory regulation or exceeds the permitted use, you will need to obtain permission directly from the copyright holder. To view a copy of this license, visit http://creativecommons.org/licenses/by/4.0/. 
DNA methylation but also by demethylation reactions. In Arabidopsis, several DNA glycosylase/lyase enzymes critical for active DNA demethylation were identified, such as DEMETER (DME), REPRESSOR OF SILENCING 1 (ROS1), DEMETER-LIKE 2 (DML2) and DML3 ${ }^{10,11}$. Recent studies have shown that the DNA methylation level is altered under a range of abiotic stresses, including $\mathrm{HM}$ and drought stresses ${ }^{12,13}$. In rice, most DNA methylation-modified genes are transcriptionally changed under cadmium $(\mathrm{Cd})$ stress, suggesting that complex DNA methylation patterns have a direct relationship with stress responses and ultimately influence gene expres$\operatorname{sion}^{12}$. However, little information on genome-wide HMinduced DNA methylation patterns and features correlated with gene expression is available for radish.

Recent studies demonstrated that exogenous salicylic acid (SA), ethylene (ET), and melatonin could alleviate the adverse effects of HMs by reducing ROS and promoting $\mathrm{HM}$ detoxification ${ }^{14-16}$. Melatonin (N-acetyl-5-methoxytryptamine, MT) has emerged as a widespread and pleiotropic organic compound in various plants, such as Arabidopsis, cabbage, and cucumber ${ }^{17-19}$. MT plays vital roles in the response to HM stresses at the physiological and biochemical levels in plants ${ }^{15,20}$. For example, exogenous MT led to an increased tolerance to salt in rice and relieved $\mathrm{Cd}$-induced damage in algae $\mathrm{e}^{21,22}$. Nevertheless, the alteration patterns of $\mathrm{Pb}$-induced DNA methylation and gene expression under MT treatment remain to be investigated in root vegetable crops, especially in radish.

Radish (Raphanus sativus L., $2 n=2 x=18$ ) is an important annual or biennial root vegetable crop of the Brassicaceae family. Plant roots are considered as the most vulnerable tissue, with a direct correlation with the uptake of HMs from soil solution. It was reported that radish roots and hypocotyls accumulated a large amount of $\mathrm{Pb}$, especially in roots, which accounted for almost $50 \%$ of the total lead content of the plant ${ }^{23,24}$. Hence, it is of great importance to reduce the $\mathrm{Pb}$ content and dissect the molecular mechanism of $\mathrm{Pb}$ detoxification in radish. Although several $\mathrm{Pb}$-induced differentially expressed genes and microRNAs have been identified ${ }^{25,26}$, no systematic studies regarding MT-mediated DNA methylation changes under $\mathrm{Pb}$ stress have been conducted in radish. In this study, MT-induced dynamic changes in $\mathrm{Pb}$ content, plant growth, and antioxidant enzyme activities were measured. Whole-genome bisulfite sequencing (WGBS) was employed to generate genome-wide cytosine methylation maps with high coverage in radish. The purposes of this study were to explore the methylation change patterns and the relationship between the methylome and gene expression changes after $\mathrm{Pb}$ stress and MT treatment in radish. Furthermore, MT-induced differentially expressed genes (DEGs) and differentially methylated region (DMR)-associated genes under $\mathrm{Pb}$ stress were identified, and an MT-mediated regulatory network of $\mathrm{Pb}$ accumulation and detoxification was proposed. These results provide fundamental insights into the molecular mechanisms underlying MT-mediated $\mathrm{Pb}$ accumulation and detoxification in root vegetable crops.

\section{Results \\ MT-induced reduction in $\mathrm{Pb}$ content in radish}

To characterize the roles of MT under Pb stress, the $\mathrm{Pb}$ contents and antioxidant enzymes of radish roots and leaves treated with $\mathrm{Pb}\left(\mathrm{NO}_{3}\right)_{2}\left(200 \mathrm{mg} \mathrm{L}^{-1}\right)$ (Pb200) or $\mathrm{Pb} 200$ plus MT $(0,10,25,50,100$ and $150 \mu \mathrm{M})$ were assayed (Fig. 1). The Pb contents of both leaves and roots were significantly decreased after MT treatment, especially in Pb200 plus $50 \mu \mathrm{M}$ MT (Pb_50MT) (Fig. 1a). However, the $\mathrm{Pb}$ content under the treatments with different MT concentrations was higher than that under the $\mathrm{Pb}$-free treatment (Control). Moreover, the weights of the roots and leaves under $\mathrm{Pb}$ 50MT were larger than those under $\mathrm{Pb} 200$, and the activities of antioxidant enzymes including ascorbate peroxidase (APX) and glutathione reductase $(\mathrm{GR})$ were increased under $\mathrm{Pb}$ stress at different melatonin concentrations, both of which reached the maximum level at $50 \mu \mathrm{M}$ melatonin, indicating that MT treatment relieved the toxicity of $\mathrm{Pb}$ stress in both the leaves and roots of radish plants (Fig. 1b-e).

\section{Features of the radish DNA methylome under $\mathrm{Pb}$ and $\mathrm{MT}$ treatment}

To explore the roles of MT in the reduction of the $\mathrm{Pb}$ content, the dynamic patterns of DNA methylation among the three treatments (Control, Pb200 and $\mathrm{Pb}$ _50MT) were characterized by whole-genome bisulfite sequencing (WGBS) (Supplementary Table S1). The percentage of methylated cytosines ( $\mathrm{mCs}$ ) showed that the methylation level increased under $\mathrm{Pb}$ treatment, and a subsequent slight decrease was observed under MT treatment. The average genome-wide methylation level of Control was $70.47 \%, 36.44 \%$ and $9.4 \%$ in the CG, CHG and $\mathrm{CHH}$ contexts, respectively. Compared with that under Pb200, the methylation level in CG, CHG and CHH under $\mathrm{Pb} \_50$ MT was reduced by $\sim 5.09 \%, 3.55 \%$ and $0.51 \%$, respectively (Fig. 2a, Supplementary Table S1). Among the Control, Pb200 and Pb_50MT libraries, the proportion of $\mathrm{mCs}$ in the CG context ( 45.43\%, 45.10\% and 44.14\%) was much higher than that in CHG $(\sim 26.10 \%, 26.16 \%$ and $26.25 \%)$ and $\mathrm{CHH}(\sim 28.47 \%$, $28.74 \%$ and $29.60 \%$ ) (Fig. 2b-d).

From the chromosome-level perspective, heterochromatic regions with a high density of transposable elements (TEs) were strongly methylated, which revealed that the hypermethylation level in repeat regions might be responsible for the repression of active transposons. 

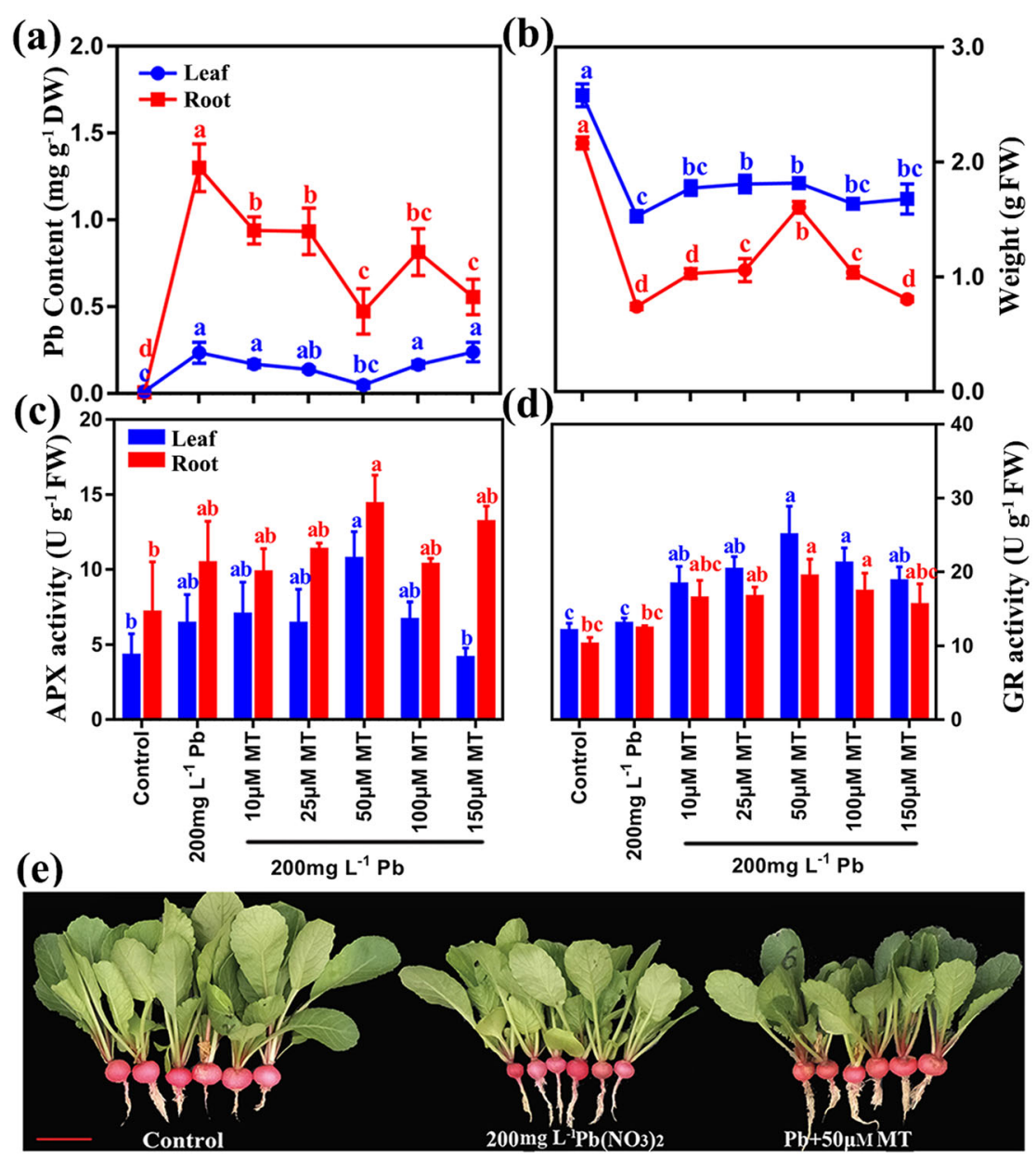

Fig. 1 Effects of MT treatment on Pb stress in radish. a Changes in Pb contents ( $\mathrm{mg} \mathrm{g}^{-1} \mathrm{DW}$ ), DW: dry weight. $\mathbf{b}$ Weight of roots and leaves under different concentrations of MT treatment, FW: fresh weight. c, d Melatonin-induced changes in the activity of ascorbate peroxidase (APX) and glutathione reductase (GR). e Growth conditions of radish under Pb-free (Control), Pb stress (Pb200), and $50 \mu \mathrm{M}$ MT treatment under Pb stress (Pb_50MT). Columns with different letters indicate significant differences at $P<0.05$ according to Duncan's multiple range test

However, a reduced methylation level was characterized in gene-rich euchromatic regions under all three treatments (Figs. 2e and S1). The methylation levels of each sequence context were high at the repeat body region and rapidly decreased in the flanking $2 \mathrm{~kb}$ regions, whereas the gene body region exhibited lower methylation levels than upstream and downstream regions, which was in accordance with findings in Arabidopsis ${ }^{27}$ (Fig. 2f, g).

\section{MT treatment decreases DNA methylation under $\mathrm{Pb}$ stress}

As shown in Fig. 2, the genomic methylation level under $\mathrm{Pb} 200$ was higher than that under Control and Pb_50MT. Hence, the differentially methylated cytosines (DMCs) and regions (DMRs) among the three treatments were analyzed to characterize the variations in methylation levels. In total, 11930, 11485 and 13100 DMRs were identified in Pb200 vs Control, Pb_50MT vs Pb200 and $\mathrm{Pb} \_50 \mathrm{MT}$ vs Control by comparing the methylomes, respectively. In the $\mathrm{Pb} 200$ vs Control group, the number of hyper-DMCs was larger than that of hypo-DMCs, similar to the number of DMRs in all three contexts. However, there were more hypo-DMC and hypo-DMR numbers in the CG and CHG contexts in Pb_50MT vs $\mathrm{Pb} 200$ (Fig. 3a). To explore the potential roles of MT treatment in DNA demethylation under Pb stress, $\mathrm{Pb} 200$ vs Control hyper-DMRs and Pb_50MT vs Pb200 hypoDMRs were used for further analysis. The methylation level of most Pb200 vs Control hyper-DMRs was lower in $\mathrm{Pb} 550 \mathrm{MT}$ than in Pb200, and the DNA methylation level of hypo-DMRs between Pb_50MT and Pb200 was lower in Control than in $\mathrm{Pb} 200$, indicating that the increased hypermethylation level under $\mathrm{Pb}$ stress might change in favor of hypomethylation after MT treatment (Figs. 3b and S2a-c). Furthermore, 1028 (34.6\%) hyper-DMRassociated genes of $\mathrm{Pb} 200$ vs Control overlapped with hypo-DMR-associated genes of Pb_50MT vs Pb200 (Figs. 

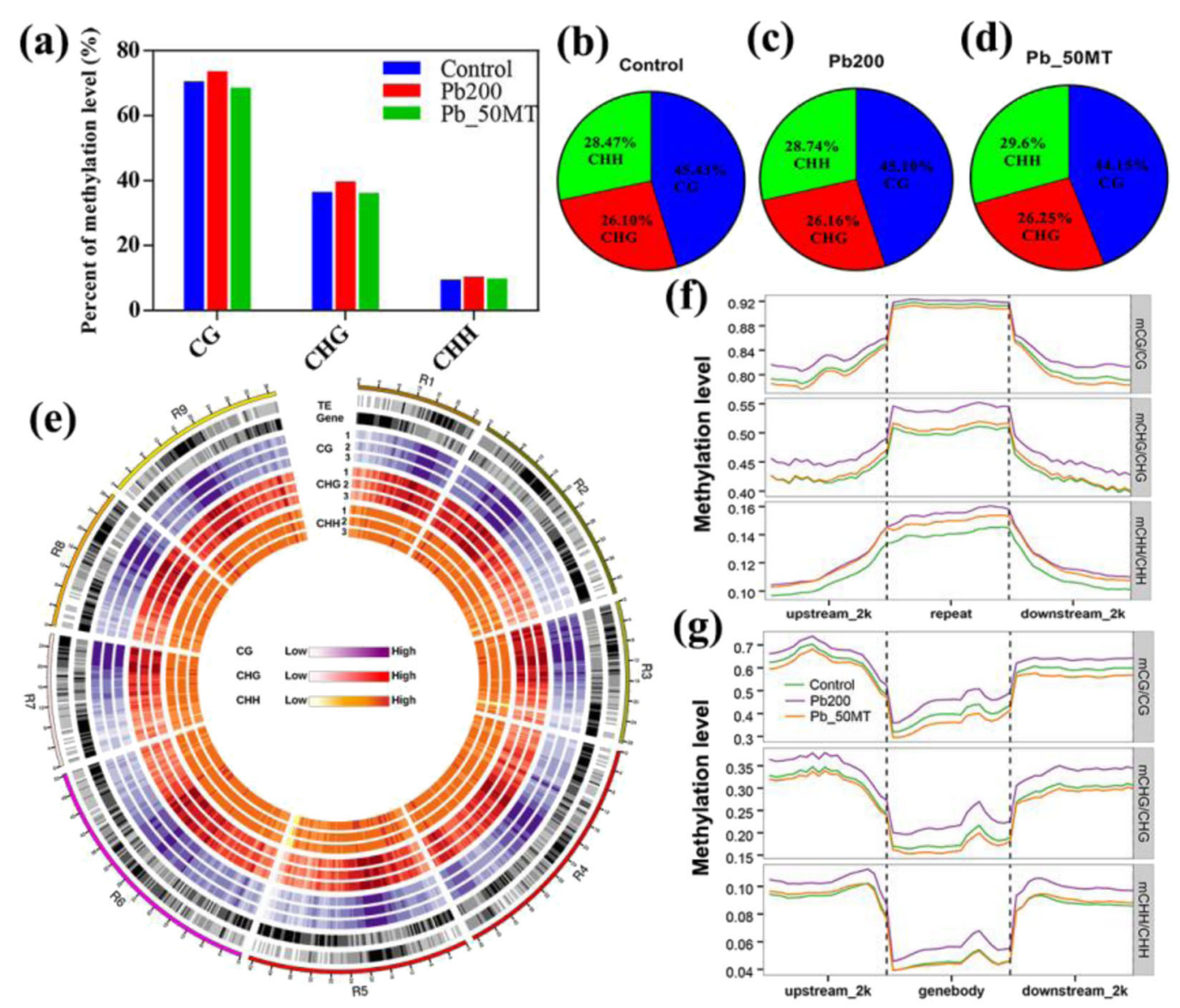

Fig. 2 Radish single-base resolution maps and features of DNA methylation. a Global levels of radish DNA methylation in three contexts. b-d Relative proportions of $\mathrm{mCs}$ in three sequence contexts $(\mathrm{CG}, \mathrm{CHG}$, and $\mathrm{CHH})$ in radish, respectively. e The landscape of DNA methylation in nine chromosomes of radish. From outer to inner: TE density, gene density and methylation of CG, CHG and CHH (1: Control, 2: Pb200, 3: Pb_50MT). Black indicates high gene/TE density. $\mathbf{f}$ DNA methylation patterns of the gene body and flanking regions in the $\mathrm{CG}, \mathrm{CHG}$ and $\mathrm{CHH}$ sequence contexts. $\mathbf{g}$ DNA methylation patterns of repeat and flanking regions in the CG, CHG and CHH sequence contexts. Upstream_2 kb indicates the $2000 \mathrm{bp}$ upstream from transcriptional start sites (TSSs), and downstream_2 kb indicates the 2000 bp downstream from transcriptional end sites (TESs)

S2d and S3a). The differential expression level of all DMR-associated genes in Pb200 vs Control was negatively related to that in Pb_50MT vs Pb200, and the overlapping DMR-associated genes exhibited a higher correlation (Fig. $\mathrm{S} 3 \mathrm{~b})$.

Based on the number of hyper/hypo-DMRs, the genomic distribution of hyper and hypo-DMRs showed that DMRs of the CG context were mainly enriched at promoter and exon regions among all three groups, while the proportion of exons was decreased with increasing repeats in both $\mathrm{mCHG}$ and $\mathrm{mCHH}$ DMRs. In particular, hyperDMRs of $\mathrm{mCHG}$ and $\mathrm{mCHH}$ were enriched at promoter and repeat regions (Fig. S3c). To investigate the dynamic changes in methylation levels in promoter and gene body regions, differential methylation analysis of $\mathrm{Pb} 200$ vs Control and Pb_50MT vs Pb200 was performed. In total, 1785 and 3650 differentially methylated genes associated with gene body and promoter regions were obtained in
$\mathrm{Pb} 200$ vs Control, while 1828 and 3593 genes harboring DMRs were identified in Pb_50MT vs Pb200 at the gene body and promoter regions, respectively. These DMRassociated genes were classified into three classes according to their location, in which the differential methylation and expression levels of these genes between two groups were negatively correlated. Moreover, the correlation coefficient showed that MT may change gene expression by altering the methylation levels of promoter and gene body regions together (Fig. 3c).

\section{Correlation between DNA methylation and gene expression under $\mathrm{Pb}$ stress}

To further investigate whether MT-induced DNA demethylation is associated with changes in gene expression, transcriptome profiles of three treatments and differential expression analysis were performed. In all, 2309 (1069 up- and 1240 downregulated), 2689 (1490 up- 
(a)

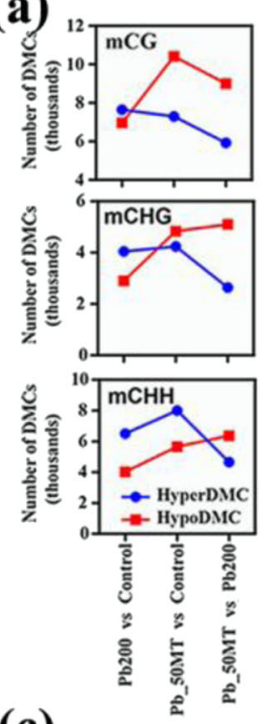

(c)

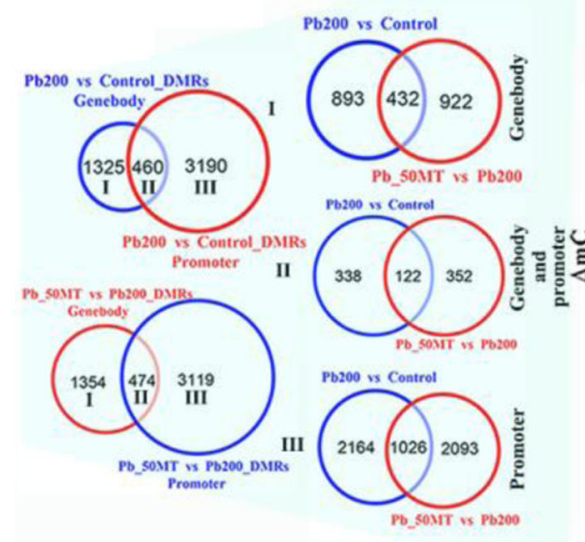

(b)

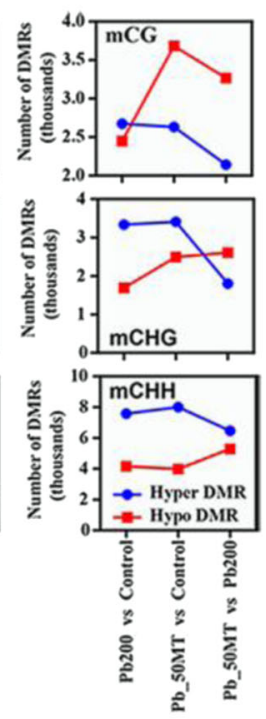

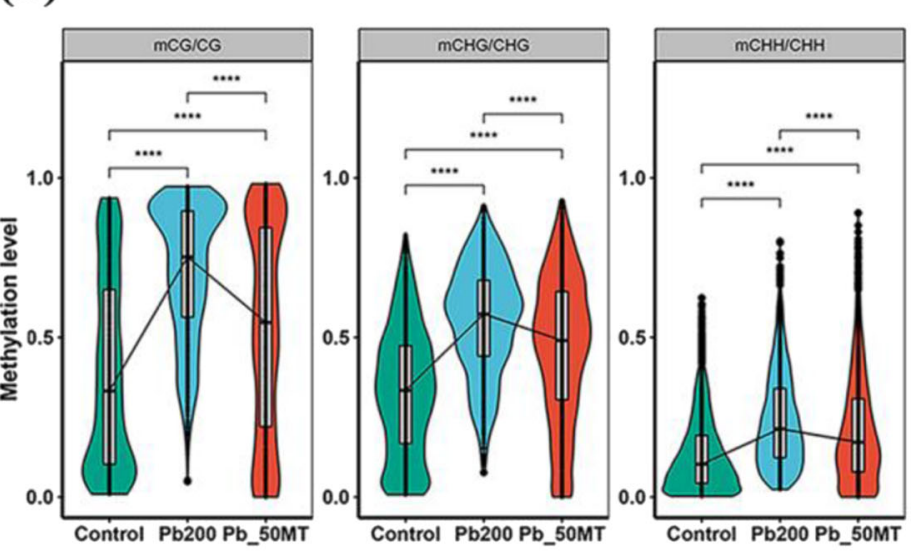
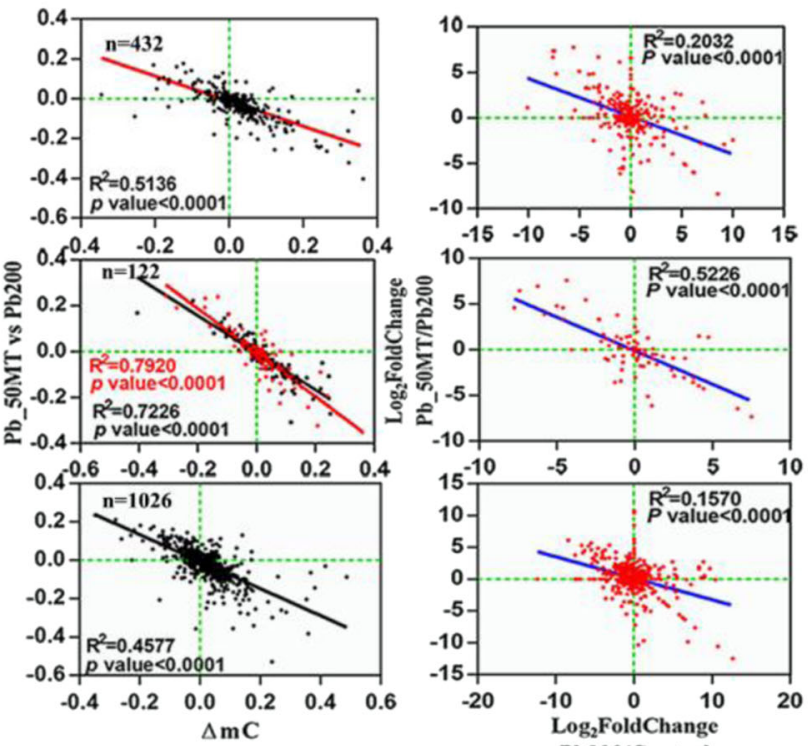

Pb200 vs Control

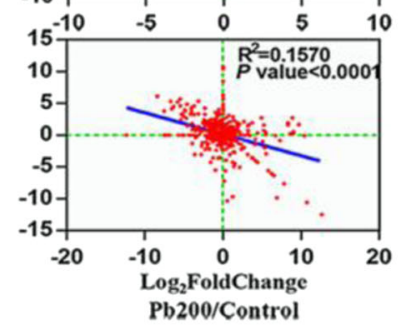

Fig. 3 MT-induced demethylation under Pb stress in radish. a Numbers of DMCs and DMRs in Pb200 vs Control, Pb_50MT vs Control, and Pb_50MT vs Pb200 are shown for the $\mathrm{mCG}, \mathrm{mCHG}$, and $\mathrm{mCHH}$ sequence contexts. $\mathbf{b}$ The DNA methylation distribution of Pb200 vs Control hyperDMRs in Pb_50MT for the $\mathrm{mCG}, \mathrm{mCHG}$ and $\mathrm{mCHH}$ sequence contexts. c The correlation of differential DNA methylation and expression levels of overlapping DMR-associated genes between Pb200 vs Control and Pb_50MT vs Pb200. Statistical significance was determined by paired two-tailed Student's $t$ test, ${ }^{*} P<0.05 ;{ }^{* *} P<0.01 ;{ }^{* *} P<0.001$; ns not significant

and 1199 downregulated), and 1342 (865 up- and 477 downregulated) differentially expressed genes (DEGs) were obtained for Pb200 vs Control, Pb_50MT vs Control, and $\mathrm{Pb}$ 50MT vs $\mathrm{Pb} 200$, respectively (Fig. 4a). The expression analysis of downregulated DEGs in Pb200 vs Control and upregulated DEGs in Pb_50MT vs Pb200 indicated that most genes with low expression in $\mathrm{Pb} 200$ showed an upregulated expression pattern in Control and Pb_50MT (Fig. 4b). Interestingly, most of the shared DEGs between Pb200 vs Control and Pb_50MT vs Control showed a similar expression trend, suggesting that these DEGs may play key roles in the biological process of the $\mathrm{Pb}$ stress response (Fig. 4c). However, a majority of overlapping DEGs between Pb200 vs Control and Pb_50MT vs Pb200 that exhibited distinct expression patterns may be regulated by $\mathrm{MT}$ to reduce $\mathrm{Pb}$ toxicity (Fig. 4d). In addition, the differential methylation level of shared DEGs between Pb200 vs Control and Pb_50MT vs Control exhibited a significantly positive correlation, while the overlapping DEGs between Pb200 vs Control and Pb_50MT vs Pb200 showed a markedly negative correlation in differential methylation levels, which were 

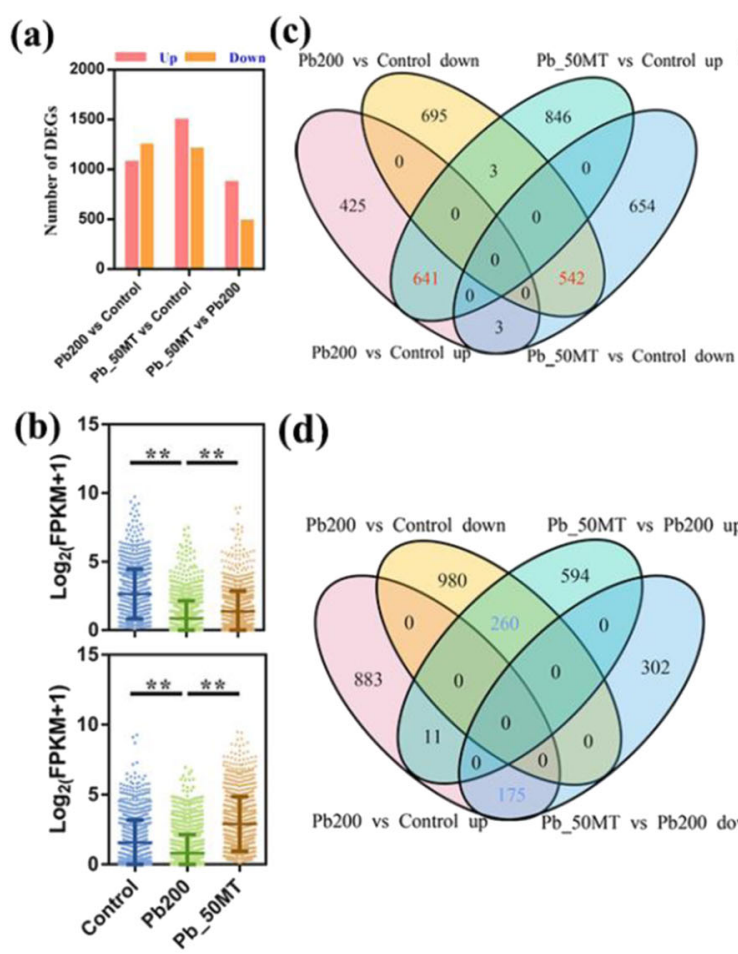

(d)
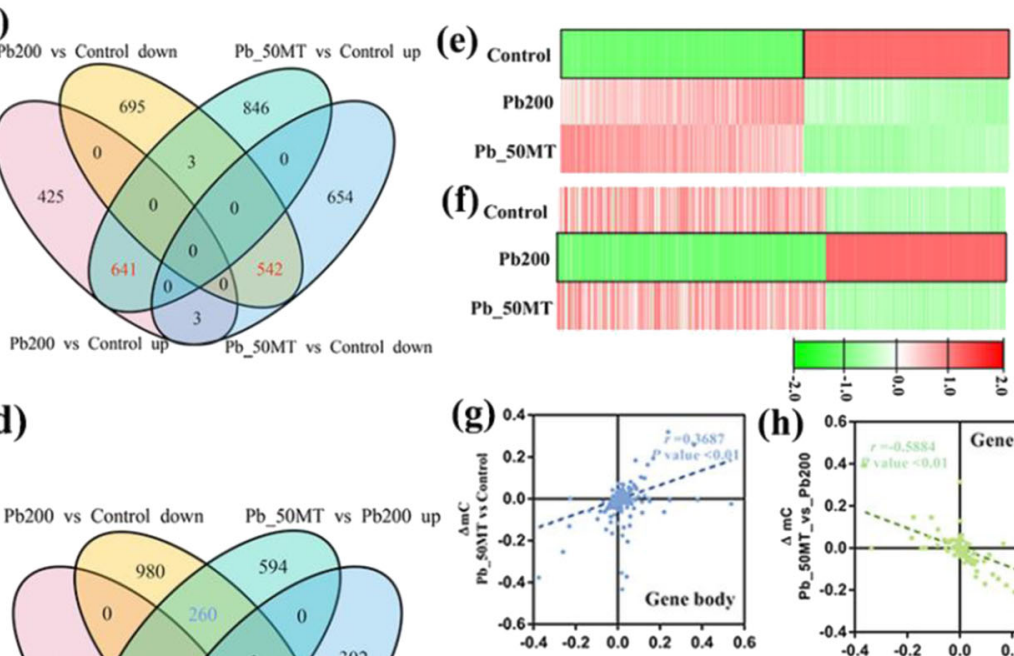

(h)
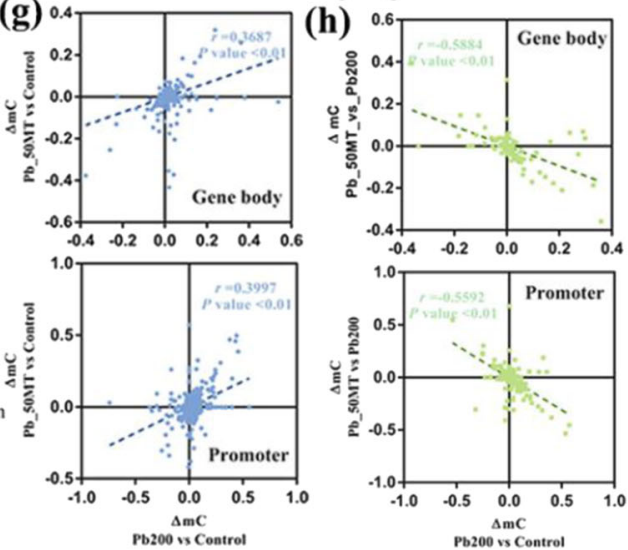

Fig. 4 Expression and methylation patterns of MT-induced DEGs. a The numbers of up- and downregulated DEGs in Pb200 vs Control, Pb_50MT vs Control, and Pb_50MT vs Pb200. b The expression patterns of Pb200 vs Control downregulated and Pb_50MT vs Pb200 upregulated DEGs. c The overlapping DEGs between Pb200 vs Control and Pb_50MT vs Control. d The overlapping DEGs between Pb200 vs Control and Pb_50MT vs Pb200. e, $\mathbf{f}$ The expression patterns of overlapped DEGs. $\mathbf{g}, \mathbf{h}$ Correlation of differential methylation levels of overlapping DEGs

consistent with the changes in gene expression (Fig. $4 \mathrm{e}-\mathrm{h})$.

Additionally, differentially expressed and methylated region-associated genes (DEGs and DMR-associated genes) overlapped and were used to explore the potential expression levels of MT-induced methylation changes associated with Pb stress. A total of 76 hyper-DMR- and 38 hypo-DMR-associated genes were significantly downregulated, and 72 hyper-DMR- and 42 hypo-DMRassociated genes showed significantly upregulated expression in $\mathrm{Pb} 200$ vs Control (Fig. 5a). Similarly, 16 downregulated and 41 upregulated DEGs were hypermethylated, while 18 downregulated and 38 upregulated DEGs were hypomethylated in Pb_50MT vs Pb200 (Fig. $5 \mathrm{~b})$. These results suggested that the methylation changes partially affected the transcriptional alterations of several DEGs with no differential transcript abundance.

Furthermore, the expression levels of all $\mathrm{Pb} 200$ vs Control hyper-DMR-associated genes and Pb_50MT vs $\mathrm{Pb} 200$ hypo-DMR-associated genes were analyzed because MT mainly decreased the methylation level (Figs. S2d and S3a). Among 4506 DMR-associated genes, 2003 were expressed in at least one sample $(\mathrm{FPKM}>1)$. The expression change was analyzed for each gene in these two groups ( $\log _{2} \mathrm{~Pb} 200 /$ Control and $\log _{2} \mathrm{~Pb} 550 \mathrm{MT} /$ $\mathrm{Pb} 200$ ), and all genes were assigned into four clusters as follows: cluster 1: 74 genes with similar expression changes in two groups, cluster 2: 53 genes that were upregulated in $\mathrm{Pb} 200$ vs Control and downregulated in $\mathrm{Pb}$ 50MT vs Pb200, cluster 3: 155 genes that were downregulated in $\mathrm{Pb} 200$ vs Control and upregulated in $\mathrm{Pb}$ 50MT vs $\mathrm{Pb} 200$, and cluster 4: 1721 genes that were not significantly changed (Fig. 5c). Interestingly, the methylation levels of cluster 1-3 genes exhibited significant differences in gene bodies but not in promoters compared with genes in cluster 4, indicating that the changes in DNA methylation in the gene body were insufficient to lead to alterations in gene expression among these genes in cluster 4 (Fig. 5d). As shown in Fig. $5 \mathrm{e}$, visualization of the methylation level for DMRassociated genes in clusters 1-4 was performed. The methylation change of an HM transport detoxification domain-containing protein (Rs204630), which was hypermethylated in Pb200 compared with the Control and Pb_50MT groups, are also presented. These results supported a critical role of MT in DNA demethylation with changes in gene expression under $\mathrm{Pb}$ stress in radish. 
(a)

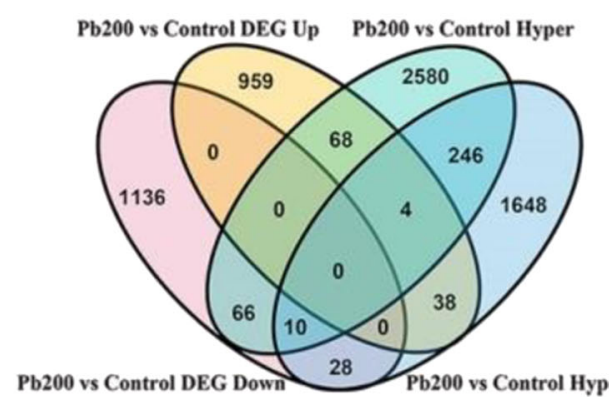

(b)

Pb_50MT vs Pb200 DEG Up Pb_50MT vs Pb200 Hyper

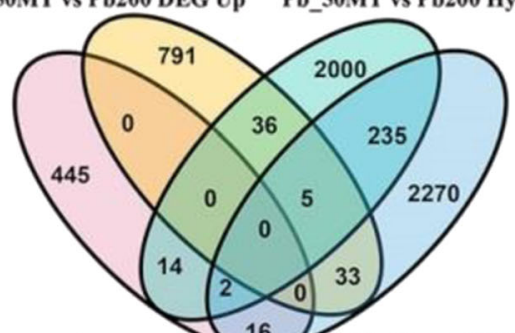

Pb_50MT vs Pb200 DEG Down 16 Pb_50MT vs Pb200 Hypo

(e)

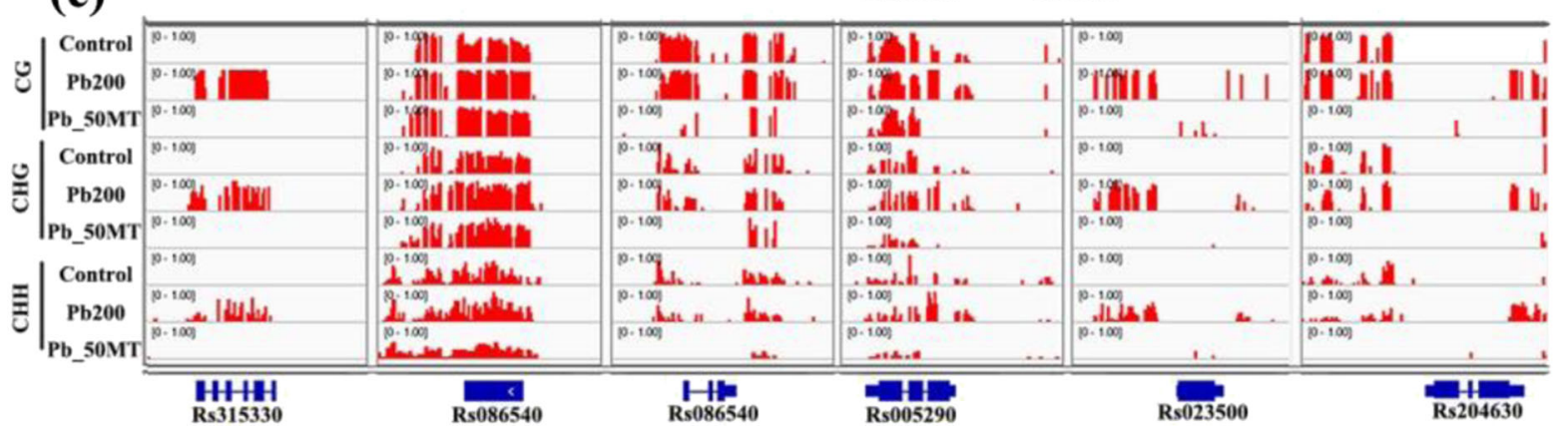

Fig. 5 The expression and methylation changes in $\mathrm{DMR}$-associated genes after $\mathrm{Pb}\left(\mathrm{NO}_{3}\right)_{2}$ and $\mathrm{MT}$ treatment in radish. $\mathbf{a}, \mathbf{b}$ The overlap between DMR-associated genes and differentially expressed genes (DEGs) in (a) Pb200 vs Control and (b) Pb_50MT vs Pb200. c Cluster of expression and methylation of Pb200 vs Control hyper-DMR-associated genes and Pb_50MT vs Pb200 hypo-DMR-associated genes. $\mathbf{d}$ Comparison of methylation levels between Clusters 1-3 and Cluster 4 in promoters and gene bodies. e IGV screenshots of six representative hyper and hypomethylated genes in the Control, Pb200, and Pb_50MT groups

\section{Functional annotation of DEGs and methylation analysis of HM-associated genes}

To understand the potential role of MT-induced differential expression and DNA demethylation, Gene Ontology (GO) analysis was performed. The GO analysis of DMR-associated genes showed that cell well biogenesis, coenzyme metabolic process and single-multicellular organism process in the biological process category as well as peptide binding and amide binding in the function category were significantly enriched terms in both $\mathrm{Pb} 200$ vs Control and Pb_50MT vs Pb200. However, the majority of GO terms, including calcium ion transport, vitamin transport and sulfur compound binding, were unique in $\mathrm{Pb} 200$ vs Control (Fig. 6a). In addition, uniquely enriched GO terms associated with ion binding were found in Pb_50MT vs Pb200, such as cation binding, iron ion binding and transition metal ion binding (Fig. 6b). These ion binding-associated genes (promoter and gene body regions) were mainly hypermethylated in $\mathrm{Pb} 200 \mathrm{vs}$ Control, while the majority of them were hypomethylated in Pb_50MT vs Pb200 (Fig. S4a). Similarly, most of the downregulated genes in $\mathrm{Pb} 200$ vs Control showed 


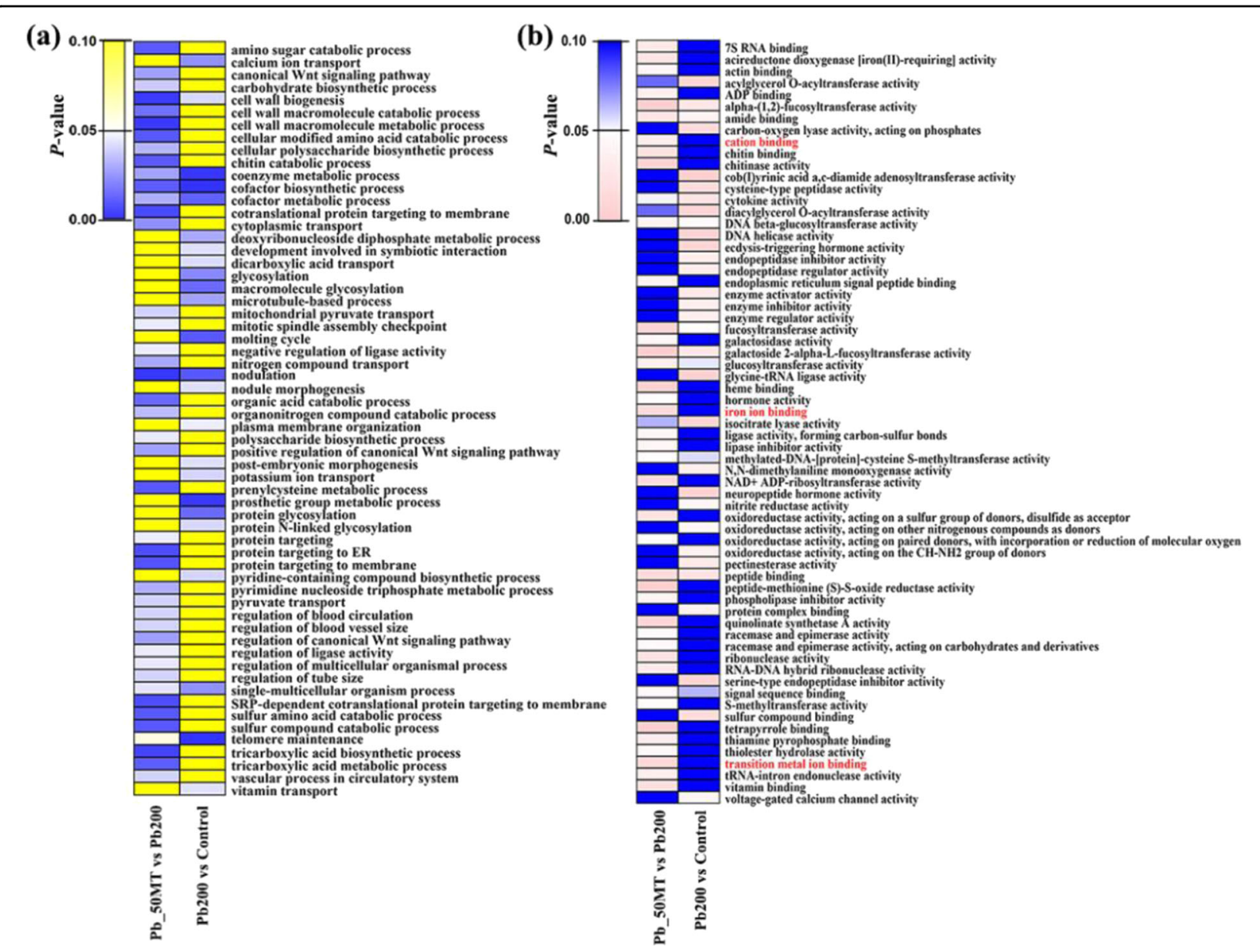

Fig. 6 GO enrichment terms of DMR-associated genes in Pb200 vs Control and Pb_50MT vs Pb200. a GO biological process terms enriched in DMR-associated genes of Pb200 vs Control and Pb_50MT vs Pb200. b GO molecular function terms enriched in DMR-associated genes of Pb200 vs Control and Pb_50MT vs Pb200

upregulated expression in Pb_50MT vs Pb200 (Fig. S4b). In addition, the significantly enriched GO terms of DEGs in the three comparisons were further investigated. Both Pb200 vs Control downregulated DEGs and Pb_50MT vs $\mathrm{Pb} 200$ upregulated DEGs were significantly enriched in response to oxidative stress, oxidation-reduction process and antioxidant activity (corrected $p$-value $<0.05$ ), suggesting that these genes might be involved in MT-induced $\mathrm{Pb}$ detoxification by enhancing antioxidant enzyme activity (Fig. S5). Furthermore, downregulated DEGs of $\mathrm{Pb}$ _50MT vs Control, consisting of 16 differentially expressed multidrug and toxic compound extrusion (MATE) genes, were mainly enriched in oxidoreductase activity and drug transporter activity.

A total of 63 DEGs associated with metal transport were identified to be involved in $\mathrm{Pb}$ reduction and detoxification. Eight out of $12 \mathrm{ABC}$ transporters showed higher expression levels in Control than in Pb_50MT, while 12 out of 16 MATE efflux proteins were upregulated under MT treatment. Additionally, 23 and 17 DEGs, including ABC transporter, heavy metal transport protein and MATE efflux protein, were upregulated with DNA methylation changes in Pb_50MT vs Pb200 and Pb200 vs Control, respectively (Figs. S6 and 7a). Interestingly, differentially methylated TFs involved in HM stresses were identified in this study, and the number of MYB and WRKY TFs accounted for a large proportion of these, which may further affect the expression of metal transporter genes (Figs. $7 \mathrm{a}$ and S7a). RT-qPCR analysis showed that RsPDR8, RsPDR12 and RsCTP were obviously upregulated under $\mathrm{Pb}$ and MT treatment. Moreover, the RsDME and RsROS1 genes, which can prevent DNA methylation, were significantly upregulated in $\mathrm{Pb}$ 50MT compared with $\mathrm{Pb} 200$, indicating that they may have a large effect on MT-induced DNA demethylation (Fig. 7b). These results suggested that MT-induced DNA demethylation, with the involvement of RsROS1 and RsDME, might alter the expression of $\mathrm{HM}$-associated genes and TFs and lead to $\mathrm{Pb}$ reduction and detoxification. 
(a)
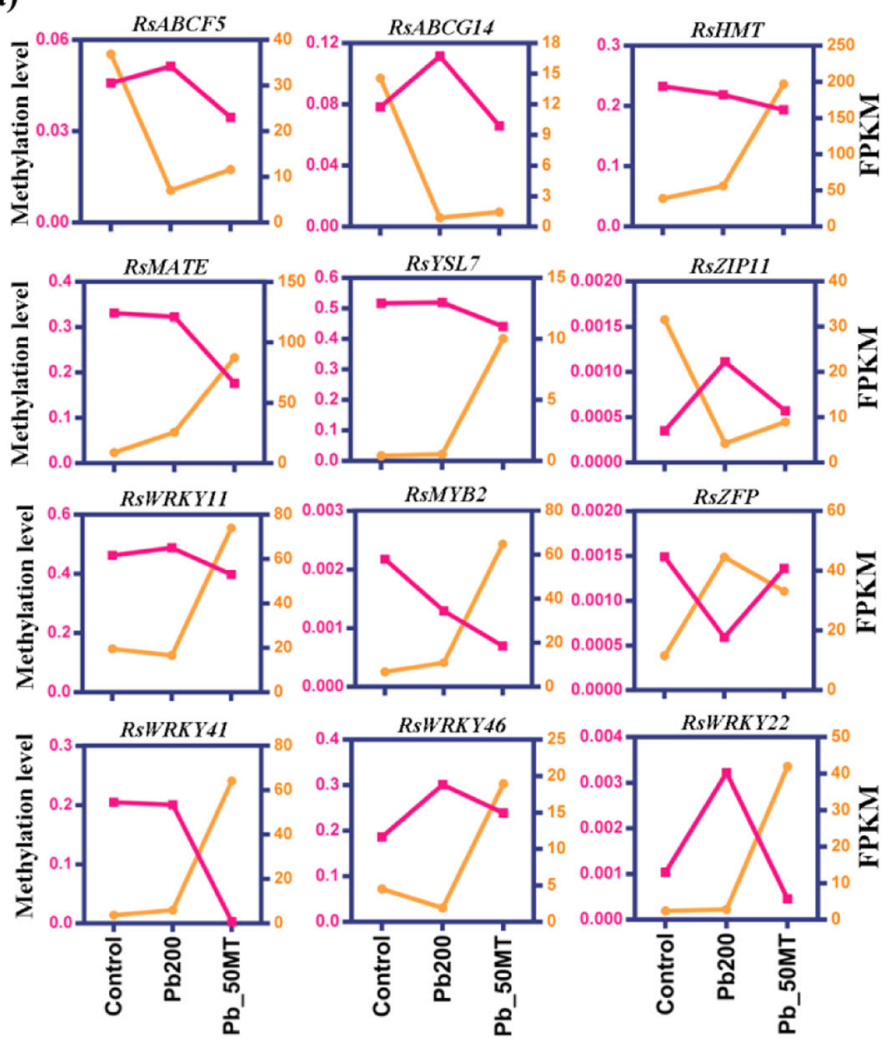

(b)

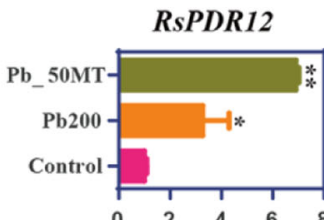

RSPDR8
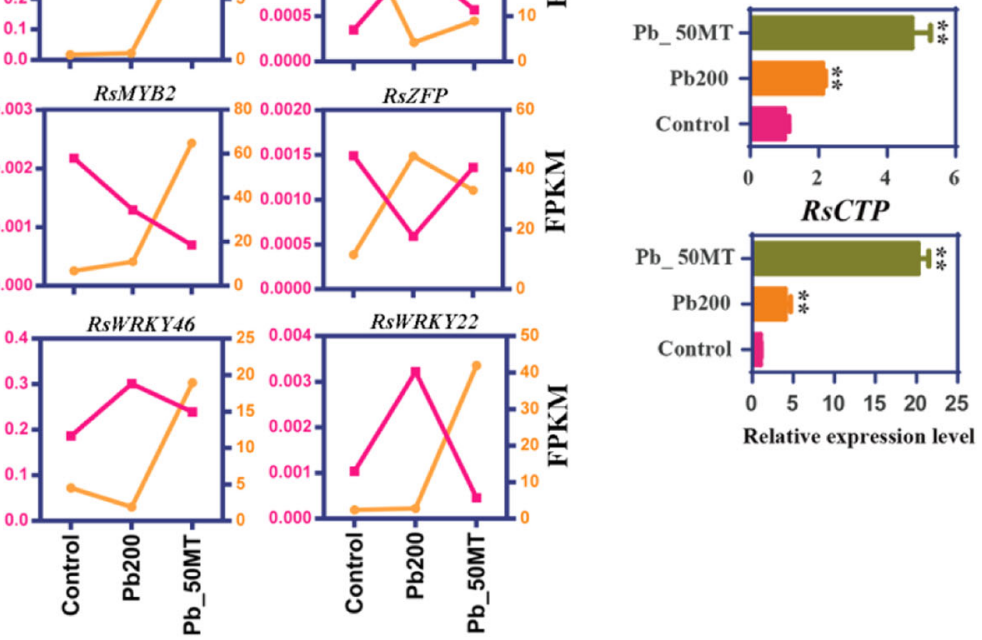

Relative expression level

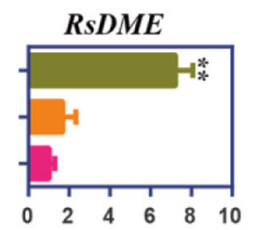

RsROS1.1

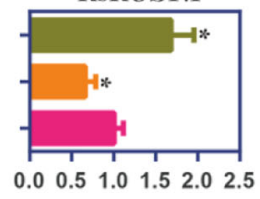

RsROS1.2

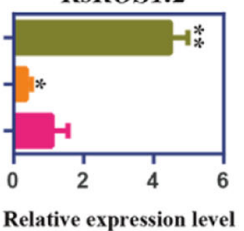

Fig. 7 Expression and methylation analysis of $\mathbf{H M}$-associated genes in response to Pb stress. a The expression and DNA methylation levels of HM-associated genes and TFs. $\mathbf{b}$ The expression levels of Pb-associated and demethylase genes under different treatments by RT-qPCR. Statistical significance was determined by paired two-tailed Student's $t$ test, ${ }^{*} P<0.05$; ${ }^{*} P<0.01$; ${ }^{* * *} P<0.001$; ns: not significant

\section{Discussion}

Lead $(\mathrm{Pb})$, one of the most widespread and dangerous metal pollutants, presents high toxicity to plants and causes serious health problems throughout the food chain $^{2}$. Melatonin (MT) has been proven to play a critical role in enhancing plant tolerance to HM stresses ${ }^{15,28}$. Radish, as a major root vegetable crop worldwide, has been demonstrated to be important in studying root HM accumulation and tolerance in root and tuberous crops ${ }^{29}$. However, systematic investigation of the DNA methylation level has not been performed in radish, especially under the application of MT. In this study, the influence of $\mathrm{Pb}$ stress and MT treatment on the association between DNA methylation and expression levels was explored with WGBS and RNA-seq technology. To our knowledge, this is the first study to investigate and characterize root methylome changes and the role of MT in decreasing $\mathrm{Pb}$ toxicity in radish.

\section{MT-mediated regulation of signal transduction and activation of antioxidant systems}

Exogenous MT treatment enhances Cd immobilization in the cell wall and vacuoles and minimizes Cd toxicity ${ }^{30}$.
In tomato, the $\mathrm{Cd}$ concentration in leaves was reduced by supplementation with exogenous $\mathrm{MT}^{31}$. Similarly, our results showed that exogenous MT application reduced $\mathrm{Pb}^{2+}$ accumulation and increased root and leaf weight (Fig. 1a, b). Diverse calcium-sensing proteins, including calmodulins (CaM), calcineurin B-like proteins (CBLs), CaM-like proteins (CML) and calcium-dependent protein kinases (CDPK), were found to be involved in heavy metal (HM) uptake, accumulation and detoxification $^{32,33}$. It was reported that ROS and CDPK played a role in $\mathrm{Pb}^{2+}$-induced cell death with the involvement of $\mathrm{Ca}^{2+}$ and triggering of mitogen-activated protein kinase (MAPK) activity ${ }^{33,34}$. In this study, 12 DEGs, encoding CaM (3), CBL (3), CML (3), CPK (1) and CDPK (2), were significantly upregulated under $\mathrm{Pb}$ stress with MT treatment. Moreover, six upregulated genes encoding MAPK and MAPKKK were found under $\mathrm{Pb}$ or $\mathrm{Pb}$ with MT treatment (Fig. S7b). These results revealed that calcium-dependent and MAPK signal transduction might be activated under $\mathrm{Pb}$ stress or $\mathrm{Pb}$ stress with $\mathrm{MT}$ treatment, which would further affect the activation of antioxidant systems and the expression of TFs and HMresponsive genes. 
Previous studies revealed that MT acted as an antioxidant combating ROS accumulation under various abiotic stresses ${ }^{21,35,36}$. In the melon response to copper stress, MT treatment increased antioxidant enzyme activities and promoted root development ${ }^{35}$. In rice shoots, MT enhanced stress tolerance through ROS scavenging and transporters caused by changes in global gene expression $^{21}$. In wheat, the MT treatment increased antioxidants and enhanced $\mathrm{Al}$ exclusion from root tips ${ }^{36}$. In our study, the antioxidant enzyme activities of APX and GR were increased in radish roots and leaves under MT treatments, which was consistent with the findings in wheat and tomato ${ }^{31,36}$ (Fig. 11c, d). Additionally, several antioxidant DEGs were markedly enriched in antioxidant activity (Fig. S5b). In detail, ascorbate peroxidase 2 (APX2) and eight of 21 peroxidases (POD52 and POD64) were obviously upregulated under MT treatment, which promoted radish plants to increase antioxidant enzyme activities to cope with $\mathrm{Pb}$ stress. Many studies have shown that glutathione S-transferases (GSTs) can accelerate HM-induced ROS scavenging by catalyzing the conjugation of GSH with HM ions into low-toxicity complexes, which are transported into the vacuole to reduce HM toxicity $^{37}$. In this study, the expression of eight DEGs encoding GST was increased under MT treatment (Fig. S8). In contrast, most of these differentially expressed antioxidant genes exhibited decreasing methylation levels. Together, these findings indicated that exogenous MT can alleviate $\mathrm{Pb}$-induced oxidative stress and achieve $\mathrm{Pb}^{2+}$ detoxification partially by regulating several redox and antioxidant genes and activating specific antioxidant systems in radish roots.

\section{MT-induced reduction of $\mathrm{Pb}$ accumulation was accompanied by methylation alteration in radish}

Currently, WGBS has been extensively used to decode single-base genome-wide methylation in plants, including Arabidopsis $^{27}$ tea $^{38}$, and apple ${ }^{13}$. In this study, global DNA methylation patterns were characterized through bisulfite sequencing, and the first single-base resolution DNA methylation map of radish was produced. In rice in response to $\mathrm{Cd}$ stress, the methylation level of genes was changed, followed by altered expression levels, and 5azacytidine promoted growth and $\mathrm{Cd}$ accumulation by enhancing the transcription of genes involved in HM transport ${ }^{12}$. Exposure to Cd elevated DNA methylation at the genome-wide level in Arabidopsis roots ${ }^{39}$. In the present study, the genome-wide DNA methylation level was increased under $\mathrm{Pb}$ stress and obviously reduced after $50 \mu \mathrm{M}$ MT treatment (Fig. 2). Interestingly, the methylation level of most hyper-DMRs in Pb200 vs Control was lower in Pb_50MT than in Pb200, while most hypo-DMRs between Pb_50MT and Pb200 exhibited higher methylation levels in Pb200 than in Control (Figs. 3a, b and
S3a-c). These results agreed with those found in grape berries, which showed that exogenous melatonin treatment decreased the methylation levels of various gene regions in the $\mathrm{CHG}$ and $\mathrm{CHH}$ contexts ${ }^{40}$. It was reported that the DNA demethylase DML2 was downregulated under cold stress in tomato, which resulted in the hypermethylation of the promoter and silencing of genes involved in the biosynthesis of flavor volatiles ${ }^{41}$. Moreover, Cd-induced lower expression of three DNA demethylase genes (ROS1, DML2 and DML3) resulted in higher DNA methylation in Arabidopsis ${ }^{39}$. In this study, RTqPCR analysis of DNA demethylase genes showed that RsROS1 was downregulated in $\mathrm{Pb} 200$ and upregulated in $\mathrm{Pb}$ 50MT, while RsDME presented higher expression levels in $\mathrm{Pb} 200$ than in Control, indicating that these genes may play a major role in decreasing the methylation level after MT treatment (Fig. 7b).

Generally, a high methylation level could inhibit gene expression, especially in the upstream regions of genes, and highly methylated genes exhibited lower expression levels ${ }^{38}$. In total, 76 and 72 hyper-DMR-associated genes were significantly down- and upregulated in $\mathrm{Pb} 200$ vs Control, respectively, indicating that DNA methylation played a vital role in suppressing expression of specific genes (Fig. 5a, b). In plants, the ion-exchangeable sites located in cell walls can be bound by $\mathrm{Pb}^{2+}$ to precipitate extracellularly $^{42}$. Hence, the metal transporter genes associated with ion binding might be responsible for metal ion uptake, translocation and detoxification. In our study, uniquely enriched GO terms, including cation binding, iron ion binding and transition metal ions, were found in $\mathrm{Pb}$-50MT vs $\mathrm{Pb} 200$, and the majority of ion binding-associated genes showing higher methylation levels in $\mathrm{Pb} 200$ were hypomethylated in Pb_50MT vs $\mathrm{Pb} 200$, suggesting that the alteration of DNA methylation by MT may play a critical role in plant response to $\mathrm{Pb}$ stress (Figs. 6b and S4).

\section{MT-mediated regulatory network of $\mathrm{Pb}$ accumulation and detoxification}

To reduce the accumulation of HMs, plants have adopted several effective strategies to restrict HM ions to the cell by binding them to the cell wall or excluding them ${ }^{43-45}$. Previous evidence has shown that several metal transporter genes, such as ATP-binding cassette $(A B C)$, zinc/iron transport protein (ZIP), multidrug and toxic compound extrusion (MATE) and HM transport protein (HMT) genes, are involved in activating the efflux of HM ions across the plasma membrane or sequestering $\mathrm{HM}$ into vacuoles to avoid toxicity ${ }^{12,46,47}$. The DNA methylation level has been considered a 'silencing' epigenetic mark and vital regulator of plant growth and stress responses through the trigger of transcriptional and posttranscriptional control of gene expression'. Previous studies showed that promoter 
methylation inhibited gene expression in the hypermethylated gene region ${ }^{48,49}$. As shown in Fig. $7 \mathrm{a}$, the methylation level of several metal transporter genes, including $A B C$ transporters (RsABCF5 and RsABCG14), RsYSL7, and RsZIP11, was decreased, while their corresponding expression level was upregulated under MT treatment (Figs. 7a and S6a). Moreover, RT-qPCR analysis showed that both the PLEIOTROPIC DRUG RESISTANCE TRANSPORTER12 (RsPDR12) and RsPDR8 genes were significantly upregulated under Pb_50MT. In Arabidopsis, AtPDR12 acts as a pump of the plasma membrane to exclude $\mathrm{Pb}^{2+}$ or $\mathrm{Pb}^{2+}$-containing toxic compounds from the cytoplasm ${ }^{50}$. AtPDR 8 was demonstrated to exclude $\mathrm{Pb}$ from epidermal cells and enhance $\mathrm{Pb}$ resistance in Arabidopsis ${ }^{51}$. These results indicated that the differential expression of these specific lowmethylated metal transporter genes might contribute to reducing $\mathrm{MT}$-induced $\mathrm{Pb}$ accumulation in radish plants.

It has been reported that several TFs, including MYB, WRKY, and zinc-finger domain-containing protein (ZFP) genes, are induced under HM stresses ${ }^{44,52}$. AtWRKYY13 directly activates the expression of the AtPDR8 gene to reduce $\mathrm{Cd}$ accumulation ${ }^{44}$. In addition, AtMYB49 directly regulates the expression of the AtbHLH38, AtbHLH101, AtHIPP22 and AtHIPP44 genes to positively control Cd accumulation in Arabidopsis ${ }^{52}$. In this study, RsMYB2 and four $R s W R K Y$ family genes (RsWRKY11, 22, 44 and 46) presented lower methylation levels with increased expression under MT treatment (Fig. 7a). It is reasonable to conclude that MT-induced DNA demethylation contribute to reducing $\mathrm{Pb}$ accumulation by altering the expression of several specific HM transporter genes in radish (Figs. 7, S4, S7a and 8).

\section{Conclusion}

In summary, a single-base resolution Pb-induced DNA methylation map was first constructed, and the role of MT-induced DNA demethylation was identified in radish. Antioxidant genes, including RsAPX2 and RsPOD64, which showed mostly low methylation levels, were upregulated to enhance antioxidant enzyme activities. Notably, MT-induced DMR-associated genes enriched in metal ion binding and oxidation-reduction process terms might play a critical role in the MT-mediated regulatory network of $\mathrm{Pb}$ accumulation and detoxification in radish. The methylation levels of TFs, including WRKYs and MYBs, which regulate metal transporter genes, including RsPDR12, could be changed by MT treatment. Moreover, MT-induced demethylation of several HM-responsive genes and TFs could further promote the process of $\mathrm{Pb}$ detoxification by regulating transcription levels in radish. Further functional characterization of these DEGs and their methylation features would be useful for better elucidation of the molecular mechanism underlying $\mathrm{Pb}$ stress responses in radish. These findings provide

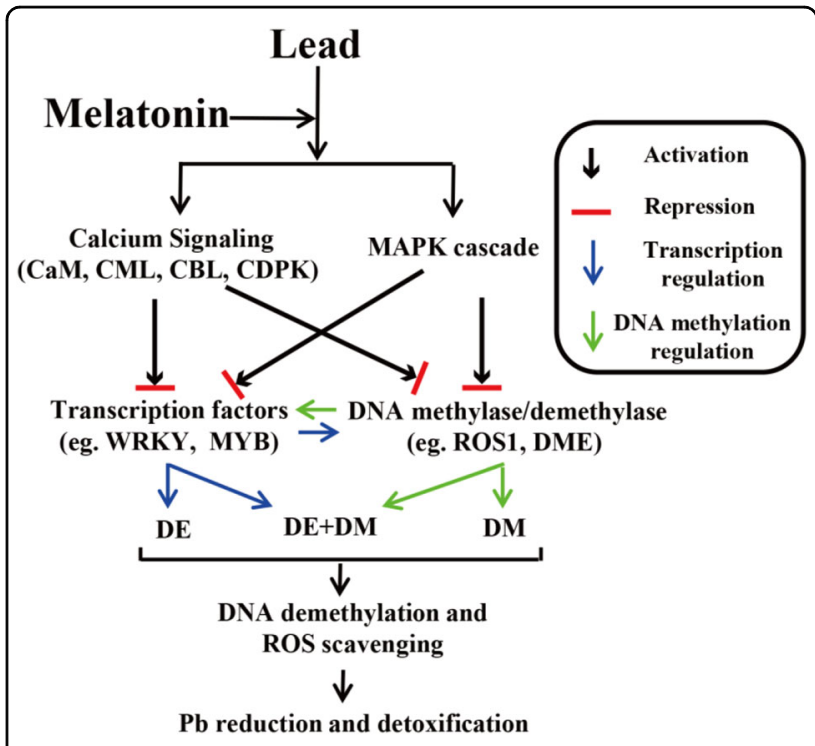

Fig. 8 A hypothetical model of MT-induced $\mathrm{Pb}$ reduction and detoxification in radish. CaM calmodulin, CML calmodulin-like protein, CBL calcineurin B-like protein, CDPK calcium-dependent protein kinase, MAPK mitogen-activated protein kinase, ROS1 REPRESSOR OF SILENCING 1, DME DEMETER, DE differential expression, DM differential methylation, ROS reactive oxygen species

fundamental insights for further investigation on $\mathrm{Pb}$ accumulation and detoxification in root vegetable crops.

\section{Materials and methods}

\section{Plant materials and growth conditions}

Radish seeds of the advanced inbred line 'NAU-YH' were surface-sterilized, rinsed, and germinated for 3 days and then cultivated in a controlled growth chamber under a photoperiod of $14 \mathrm{~h} \mathrm{light}$ at $25^{\circ} \mathrm{C}$ and $10 \mathrm{~h}$ dark at $18^{\circ} \mathrm{C}$ for three weeks. Seedlings of similar sizes were transferred into a plastic container with half-strength Hoagland nutrient solution. After 5 days, plants were treated with 0 and $200 \mathrm{mg} \mathrm{L}^{-1} \mathrm{~Pb}\left(\mathrm{NO}_{3}\right)_{2}$. Hydroponic solution was refreshed every five days. Seedlings grown in $\mathrm{Pb}$-free nutrient solution were used as controls. To determine the optimal dose of melatonin (MT) that could effectively decrease accumulation, the foliar portion of $\mathrm{Pb}$-treated radish seedlings was sprayed with different concentrations of MT $(0,10,25,50,100$ and $150 \mu \mathrm{M})$ at $8: 00 \mathrm{pm}$. Based on the preliminary dose trial, $50 \mu \mathrm{M}$ MT was selected for the rest of the experiments, considering its effect in reducing $\mathrm{Pb}$ contents. Three replicates were used for each treatment, with 6-8 plants for each replicate.

\section{Determination of antioxidant enzyme activities and $\mathrm{Pb}$ contents}

To measure the antioxidant enzyme activities, $0.1 \mathrm{~g}$ of fresh leaf and root samples was suspended in potassium phosphate buffer $\left(50 \mathrm{mmol} \mathrm{L}^{-1}, \mathrm{pH} 7.0\right)$ containing 
$0.1 \mathrm{mmol} \mathrm{L}^{-1}$ EDTA and 1\% polyvinylpyrrolidone (w/v). The fully vortexed homogenate was centrifuged for $15 \mathrm{~min}$ at $12,000 \times g\left(4{ }^{\circ} \mathrm{C}\right)$, and the supernatant was collected to detect the activities of glutathione reductase (GR) and ascorbate peroxidase (APX) enzymes according to previous studies ${ }^{53}$. For the $\mathrm{Pb}$ content, roots and leaves were separately collected and weighed, dried at $85^{\circ} \mathrm{C}$ for $24 \mathrm{~h}$, and ground into powder with a mortar. Each sample $(0.2 \mathrm{~g})$ was transferred into a highpressure polytetrafluoroethylene vessel to which $5 \mathrm{~mL}$ $\mathrm{HNO}_{3}$ was added. The vessel was sealed with a screwcap and digested by a microwave (Mars 6, CEM Technologies, USA). The digested solutions were used to determine the $\mathrm{Pb}$ content by an inductively coupled plasma optical emission spectrometer ${ }^{54}$ (ICP-OES, Thermo Fisher iCAP 7400).

\section{Construction of genomic methyl cytosine libraries and methyl-Seq analysis}

Total genomic DNA was extracted from the roots of seedlings, including $\mathrm{Pb}$-free (Control), $\mathrm{Pb}$-treated ( $\mathrm{Pb} 200)$, and $\mathrm{Pb}$ plus $50 \mu \mathrm{M}$ MT-treated (Pb_50MT) seedlings, using a Plant Genomic DNA Kit (Tiangen, Beijing, China) for whole-genome Methyl-Seq. DNA quality was checked using a NanoPhotometer ${ }^{\circledR}$ spectrophotometer (IMPLEN, CA, USA). A total of $5.2 \mu \mathrm{g}$ genomic DNA was fragmented by sonication to 200-300 bp with Covaris S220, followed by end repair and adenylation. These DNA fragments were treated twice with bisulfite using an EZ DNA MethylationGoldTM Kit (Zymo Research). The prepared library was sequenced on the Illumina HiSeq platform (Novogene, Beijing, China), and base calling was performed with the Illumina CASAVA pipeline. FastQC (fastqc_v0.11.5) was used to perform basic statistics on the quality of raw reads. Clean reads were generated by filtering out adaptor sequences, contaminants, and low-quality reads using fastp software $^{55}$ with the following parameter settings: (1) -length_required $=36 ;(2)$-cut_front_window_size $=1$; (3) -cut_front_mean_quality $=3$; (4) -cut_tail_window_size = 1 ; and (5) -cut_tail_mean_quality $=3$. Bismark software (version 0.16.3) was used to perform alignments of bisulfitetreated reads to a reference genome $e^{56,57}$.

\section{Methylation level and differentially methylated region (DMR) analyses}

To calculate the methylation levels of the sequences, a sequence was divided into multiple bins, and the bin size was $10 \mathrm{~kb}$. A sliding-window approach was applied for methylation-level analysis. With window size $=3000 \mathrm{bp}$ and step size $=600 \mathrm{bp}$, and the sum of methylated and unmethylated read counts was calculated in each window. The methylation level (ML) for each window or C site showed the fraction of methylated $\mathrm{C}$ and was defined as $\mathrm{ML}(\mathrm{C})=\operatorname{reads}(\mathrm{mC}) /[\operatorname{reads}(\mathrm{mC})+\operatorname{reads}(\mathrm{C})]^{58}$.
The Bioconductor package DSS ${ }^{59}$ (Dispersion Shrinkage for Sequencing) was used to identify differentially methylated regions (DMRs) following default parameter settings with a reduced smoothing size (smoothing span $=200$ ). According to the distribution of DMRs throughout the genome, genes related to DMRs were defined as DMR-associated genes whose gene body region (from TSS to TES) or promoter region (upstream $2 \mathrm{~kb}$ from the TSS) overlapped with DMRs.

\section{RNA sequencing and differential analysis}

A total of $3 \mu \mathrm{g}$ RNA per sample (Control, Pb200 and Pb_50MT) was used as an input material for RNA sample preparation. The libraries were sequenced on an Illumina HiSeq 2500 platform, and $150 \mathrm{bp}$ paired-end reads were generated. Paired-end clean reads were aligned to the reference genome ${ }^{56}$ using Bowtie and TopHat 2 programs $^{60,61}$. The mapped reads of each sample were assembled by StringTie (v1.3.1) in a reference-based approach $^{62}$. The gene expression levels of mRNA reads were calculated using the fragments per kilobase of transcript per million fragments mapped (FPKM) approach, and DESeq2 was used to obtain differentially expressed genes (DEGs) with fold change $>1$ and $P$-value $<0.05$.

\section{RT-qPCR analysis}

Total RNA was isolated from the control and treated radish roots using an RNA simple total RNA kit (Tiangen, Beijing, China). RNA was reverse transcribed into cDNA using a PrimeScript ${ }^{\mathrm{TM}}$ II 1st Strand cDNA Synthesis Kit (Takara, Dalian, China). RT-qPCR analysis was conducted using SYBR Green PCR Master Mix with ROX (Takara, Dalian, China). Each $20 \mu \mathrm{l}$ reaction contained $10 \mu \mathrm{l}$ of $2 \times$ SYBR Green PCR Master Mix (Takara, Dalian, China), $0.2 \mu \mathrm{M}$ of each primer, and $2 \mu \mathrm{L}$ of diluted cDNA. PCR was performed on a LightCycler ${ }^{\circledR} 480$ System (Roche, Mannheim, Germany) with the following thermal cycling conditions: $95^{\circ} \mathrm{C}$ for $3 \mathrm{~min}$ and 40 cycles of $95^{\circ} \mathrm{C}$ for $5 \mathrm{~s}$, $58^{\circ} \mathrm{C}$ for $30 \mathrm{~s}$, and $72^{\circ} \mathrm{C}$ for $10 \mathrm{~s}^{63}$. The $2^{-\Delta \Delta C_{\mathrm{T}}}$ method was used to calculate relative expression levels ${ }^{64}$. Three biological and technical replicates were performed, and the RsActin gene was employed as the internal standard ${ }^{57}$. The significance of differences between groups was evaluated using Student's $t$ test. Analyses were performed with GraphPad Prism software (GraphPad Software, San Diego, California).

\section{Acknowledgements}

This work was partially supported by grants from the National Natural Science Foundation of China (31601766), National Key Technology R\&D Program of China (2018YFD1000800), the earmarked fund for Jiangsu Agricultural Industry Technology System (JATS [2020] 410), the Jiangsu Agricultural Science and Technology Innovation Fund (CX(20)3144), and the Project Funded by the Priority Academic Program Development of Jiangsu Higher Education Institutions (PAPD). 


\section{Author contributions}

M.T. performed the data analysis and wrote the manuscript. L.X., Y.W., and M.T. designed the research. J.D., X.Z., and K.W. prepared the root samples for the experiment. M.T., J.Y., and C.L. performed data collection and interpretation. L. $X$. and L.L. helped revise of manuscript. L.L. conceived and designed the research. All authors read and approved the final manuscript.

\section{Data availability}

The datasets generated during the current study have been deposited into the National Center for Biotechnology Information under project numbers PRJNA598317 and PRJNA598307.

\section{Conflict of interest}

The authors declare no competing interests.

Supplementary information The online version contains supplementary material available at https://doi.org/10.1038/s41438-021-00561-8.

Received: 31 August 2020 Revised: 11 March 2021 Accepted: 26 March 2021

Published online: 01 June 2021

\section{References}

1. Ashraf, U. \& Tang, X. Yield and quality responses, plant metabolism and metal distribution pattern in aromatic rice under lead (Pb) toxicity. Chemosphere 176, 141-155 (2017).

2. Lal, S. et al. Exploring the survival tactics and plant growth promising traits of root-associated bacterial strains under $\mathrm{Cd}$ and $\mathrm{Pb}$ stress: a modelling based approach. Ecotoxicol. Environ. Saf. 170, 267-277 (2019).

3. Shahid, M., Pinelli, E. \& Dumat, C. Review of Pb availability and toxicity to plants in relation with metal speciation; role of synthetic and natural organic ligands. J. Hazard. Mater. 219, 1-12 (2012).

4. Sharma, S. S., Dietz, K. J. \& Mimura, T. Vacuolar compartmentalization as indispensable component of heavy metal detoxification in plants. Plant Cell Environ. 39, 1112-1126 (2016).

5. Ji, Y. et al. Heavy metal accumulation, risk assessment and integrated biomarker responses of local vegetables: a case study along the Le'an river. Chemosphere 199, 361-371 (2018).

6. Bouyer, D. et al. DNA methylation dynamics during early plant life. Genome Biol. 18, 179 (2017).

7. Ma, Y. Z. et al. Disrupted genome methylation in response to high temperature has distinct affects on microspore abortion and anther indehiscence. Plant Cell 30, 1387-1403 (2018).

8. Matzke, M. A. \& Mosher, R. A. RNA-directed DNA methylation: an epigenetic pathway of increasing complexity. Nat. Rev. Genet. 15, 394-408 (2014).

9. Law, J. A. \& Jacobsen, S. E. Establishing, maintaining and modifying DNA methylation patterns in plants and animals. Nat. Rev. Genet. 11, 204 (2010).

10. Zhu, J. K. Active DNA demethylation mediated by DNA glycosylases. Annu. Rev. Genet. 43, 143-166 (2009).

11. Zhang, H. M., Lang, Z. B. \& Zhu, J. K. Dynamics and function of DNA methylation in plants. Nat. Rev. Mol. Cell Biol. 19, 489-506 (2018).

12. Feng, S. J. et al. Variation of DNA methylation patterns associated with gene expression in rice (Oryza sativa) exposed to cadmium. Plant Cell Environ. 39, 2629-2649 (2016)

13. $\mathrm{Xu}, \mathrm{J}$. et al. Single-base methylome analysis reveals dynamic epigenomic differences associated with water deficit in apple. Plant Biotechnol. J. 16, 672-687 (2018).

14. Gu, Q. et al. Melatonin confers plant tolerance against cadmium stress via the decrease of cadmium accumulation and reestablishment of microRNAmediated redox homeostasis. Plant Sci. 261, 28-37 (2017).

15. Cai, S. Y. et al. HsfA1a upregulates melatonin biosynthesis to confer cadmium tolerance in tomato plants. J. Pineal Res. 62, e12387 (2017).

16. Sami, A. et al. Melatonin mitigates cadmium and aluminium toxicity through modulation of antioxidant potential in Brassica napus L. Plant Biol. 22, 679-690 (2020).

17. Byeon, Y., Lee, H. Y., Lee, K. \& Back, K. Caffeic acid O-methyltransferase is involved in the synthesis of melatonin by methylating $\mathrm{N}$-acetylserotonin in Arabidopsis. J. Pineal Res. 57, 219-227 (2014).
18. Aguilera, Y. et al. Estimation of scavenging capacity of melatonin and other antioxidants: contribution and evaluation in germinated seeds. Food Chem 170, 203-211 (2015).

19. Zhang, R., Sun, Y., Liu, Z., Jin, W. \& Sun, Y. Effects of melatonin on seedling growth, mineral nutrition, and nitrogen metabolism in cucumber under nitrate stress. J. Pineal Res. 62, e12403 (2017).

20. Kanwar, M. K. et al. Melatonin promotes metabolism of bisphenol A by enhancing glutathione-dependent detoxification in Solanum lycopersicum L. J. Hazard. Mater. 388, 121727 (2019).

21. Liang, C. Z. et al. Melatonin delays leaf senescence and enhances salt stress tolerance in rice. J. Pineal Res. 59, 91-101 (2015).

22. Tal, O., Haim, A., Harel, O. \& Gerchman, Y. Melatonin as an antioxidant and its semi-lunar rhythm in green macroalga Ulva sp. J. Exp. Bot. 62, 1903-1910 (2011).

23. Massaccesi, L. et al. Ligands involved in $\mathrm{Pb}$ immobilization and transport in lettuce, radish, tomato and Italian ryegrass. J. Plant Nutr. Soil Sci. 177, 766-774 (2014).

24. Wang, Y. et al. Transport, ultrastructural localization, and distribution of chemical forms of lead in radish (Raphanus sativus L.). Front. Plant Sci. 6, 293 (2015).

25. Wang, Y. et al. Identification of radish (Raphanus sativus L.) miRNAs and their target genes to explore miRNA-mediated regulatory networks in lead $(\mathrm{Pb})$ stress responses by high-throughput sequencing and degradome analysis. Plant Mol. Biol. Report. 33, 358-376 (2015).

26. Wang, Y. et al. Transcriptome profiling of radish (Raphanus sativus L.) root and identification of genes involved in response to lead (Pb) stress with next generation sequencing. PLoS One 8, e66539 (2013).

27. Cokus, S. J. et al. Shotgun bisulphite sequencing of the Arabidopsis genome reveals DNA methylation patterning. Nature 452, 215 (2008).

28. Kanwar, M. K., Yu, J. Q. \& Zhou, J. Phytomelatonin: recent advances and future prospects. J. Pineal Res. 65, e12526 (2018).

29. $\mathrm{Xu}, \mathrm{L}$. et al. Dissecting root proteome changes reveals new insight into cadmium stress response in radish (Raphanus sativus L.). Plant Cell Physiol. 58 1901-1913 (2017)

30. Hasan, M. K. et al. Melatonin mitigates cadmium phytotoxicity through modulation of phytochelatins biosynthesis, vacuolar sequestration, and antioxidant potential in Solanum lycopersicum L. Front. Plant Sci. 6, 601 (2015).

31. Li, M. Q. et al. Melatonin mediates selenium-induced tolerance to cadmium stress in tomato plants. J. Pineal Res. 61, 291-302 (2016).

32. $\mathrm{Ni}$, J. et al. Exogenous melatonin confers cadmium tolerance by counterbalancing the hydrogen peroxide homeostasis in wheat seedlings. Molecules 23, 799 (2018).

33. Jalmi, S. K. et al. Traversing the links between heavy metal stress and plant signaling. Front. Plant Sci. 9, 12 (2018).

34. Huang, T. L. \& Huang, H. J. ROS and CDPK-like kinase-mediated activation of MAP kinase in rice roots exposed to lead. Chemosphere 71, 1377-1385 (2008).

35. Hu, Z., Fu, Q., Zheng, J., Zhang, A. \& Wang, H. Transcriptomic and metabolomic analyses reveal that melatonin promotes melon root development under copper stress by inhibiting jasmonic acid biosynthesis. Hortic. Res. 7, 79 (2020).

36. Sun, $C$. et al. Melatonin ameliorates aluminum toxicity through enhancing aluminum exclusion and reestablishing redox homeostasis in roots of wheat J. Pineal Res. 68, e12642 (2020).

37. Ahsan, N., Nakamura, T. \& Komatsu, S. Differential responses of microsomal proteins and metabolites in two contrasting cadmium (Cd)-accumulating soybean cultivars under Cd stress. Amino Acids 42, 17-327 (2012).

38. Wang, L. et al. DNA methylome analysis provides evidence that the expansion of the tea genome is linked to TE bursts. Plant Biotechnol. J. 17, 826-835 (2019).

39. Fan, S. K. et al. Inhibition of DNA demethylation enhances plant tolerance to cadmium toxicity by improving iron nutrition. Plant Cell Environ. 43, 275-291 (2020).

40. Gao, S., Ma, W., Lyu, X., Cao, X. \& Yao, Y. Melatonin may increase disease resistance and flavonoid biosynthesis through effects on DNA methylation and gene expression in grape berries. BMC Plant Biol. 20, 231 (2020).

41. Zhang, B. et al. Chilling-induced tomato flavor loss is associated with altered volatile synthesis and transient changes in DNA methylation. Proc. Natl Acad. Sci. USA 113, 12580-12585 (2016).

42. Rucińska-Sobkowiak, R. et al. Water status and water diffusion transport in lupine roots exposed to lead. Environ. Exp. Bot. 87, 100-109 (2013).

43. Bali, S. et al. Role of P-type ATPase metal transporters and plant immunity induced by jasmonic acid against Lead $(\mathrm{Pb})$ toxicity in tomato. Ecotoxicol. Environ. Saf. 174, 283-294 (2019). 
44. Sheng, Y. et al. The WRKY transcription factor, WRKY13, activates PDR8 expression to positively regulate cadmium tolerance in Arabidopsis. Plant Cell Environ. 42, 891-903 (2019).

45. Hasan, M. et al. Responses of plant proteins to heavy metal stress - a review. Front. Plant Sci. 8, 1492 (2017).

46. Hwang, J. U. et al. Plant $A B C$ transporters enable many unique aspects of a terrestrial plant's lifestyle. Mol. Plant 9, 338-355 (2016).

47. Zhu, H. H. et al. Transcriptome analysis providing novel insights for $\mathrm{Cd}$-resistant tall fescue responses to Cd stress. Ecotoxicol. Environ. Saf. 160, 349-356 (2018).

48. Zemach, A. et al. Local DNA hypomethylation activates genes in rice endosperm. Proc. Natl Acad. Sci. USA 107, 18729-18734 (2010)

49. $\mathrm{Li}, \mathrm{X}$. et al. Single-base resolution maps of cultivated and wild rice methylomes and regulatory roles of DNA methylation in plant gene expression. BMC Genomics 13, 300 (2012).

50. Lee, M., Lee, K., Lee, J., Noh, E. W. \& Lee, Y. AtPDR12 contributes to lead resistance in Arabidopsis. Plant Physiol. 138, 827-836 (2005).

51. Kim, D., Bovet, L., Maeshima, M., Martinoia, E. \& Lee, Y. The ABC transporter AtPDR8 is a cadmium extrusion pump conferring heavy metal resistance. Plant J. 50, 207-218 (2007)

52. Zhang, P. et al. The R2R3-MYB transcription factor MYB49 regulates cadmium accumulation. Plant Physiol. 180, 529-542 (2019). pp.

53. Qi, Z. Y. et al. Melatonin alleviates high temperature-induced pollen abortion in Solanum lycopersicum. Molecules 23, 386 (2018).

54. Yun-Yun, C. et al. Melatonin alleviates copper toxicity via improving copper sequestration and ROS scavenging in cucumber. Plant Cell Physiol. 60, 562-574 (2019).
55. Chen, S., Zhou, Y., Chen, Y. \& Gu, J. fastp: an ultra-fast all-in-one FASTQ preprocessor. Bioinformatics 34, i884-i890 (2018).

56. Krueger, F. \& Andrews, S. R. Bismark: a flexible aligner and methylation caller for Bisulfite-Seq applications. Bioinformatics 27, 1571-1572 (2011).

57. Jeong, Y. M. et al. Elucidating the triplicated ancestral genome structure of radish based on chromosome-level comparison with the Brassica genomes. Theor. Appl. Genet. 129, 1357-1372 (2016).

58. Smallwood, S. A. et al. Single-cell genome-wide bisulfite sequencing for assessing epigenetic heterogeneity. Nat. Methods 11, 817 (2014).

59. Feng, H., Conneely, K. N. \& Wu, H. A Bayesian hierarchical model to detect differentially methylated loci from single nucleotide resolution sequencing data. Nucleic Acids Res. 8, e69 (2014).

60. Langmead, B. \& Salzberg, S. Fast gapped-read alignment with Bowtie 2. Nat Methods 9, 357-359 (2012).

61. Kim, D. et al. TopHat2: accurate alignment of transcriptomes in the presence of insertions, deletions and gene fusions. Genome Biol. 14, R36 (2013).

62. Pertea, M., Kim, D., Pertea, G. M., Leek, J. T. \& Salzberg, S. L. Transcript-level expression analysis of RNA-seq experiments with HISAT, StringTie and Ballgown. Nat. Protoc. 11, 1650-1667 (2016).

63. Fan, L. X. et al. A genome-wide association study uncovers a critical role of the RsPAP2 gene in red-skinned Raphanus sativus L. Horticulture Res. 7, 164 (2020).

64. Livak, K. J. \& Schmittgen, T. D. Analysis of relative gene expression data using real-time quantitative $P C R$ and the $2^{-\Delta \Delta C_{\mathrm{T}}}$ method. Methods 25, 402-408 (2001). 